\title{
Discurso y sujeto en las perspectivas de Pêcheux y Voloshinov ${ }^{1}$
}

\author{
Silvia Hernández, Paula Morel y Ricardo Terriles \\ (Universidad de Buenos Aires)
}

Recibido: $11 / 11 / 2010$

Aceptado: 25/1/2011

\begin{abstract}
RESUMEN: En el presente trabajo realizaremos una comparación entre los planteos de Michel Pêcheux y de Valentin Voloshinov, con el propósito de analizar de qué modo es problematizado, en forma más o menos explícita, el estatuto del sujeto en sus teorías del discurso. Específicamente nos centraremos en la cuestión del sujeto porque entendemos que allí reside uno de los nudos complicados que, aún hoy, constituyen desafíos para la teoría y el análisis del discurso.
\end{abstract}

Palabras clave: Ideología / sujeto / signo / discurso

\section{Discourse and subject in Pêcheux and Voloshinov's perspectives}

Summary: This article aims to compare the approaches of Michel Pêcheux and Valentin Voloshinov in order to analyze the way both authors focus the statute of the subject in their theories of discourse. The issue of the subject is considered one of the most important topic that, still today, challenges both theory and discourse analysis.

Key words: Ideology / subject / sign / discourse

1 Este trabajo se inscribe en el equipo de investigación UBACyT "Discurso, política, sujeto: Encuentros entre el marxismo, el psicoanálisis y las teorías de la significación", dirigido por el profesor Sergio Caletti, de la Facultad de Ciencias Sociales de la Universidad de Buenos Aires (FSOC-UBA). 


\section{Introducción y cuestiones preliminares. El concepto de ideología}

$\mathrm{P}$ roducto de épocas y contextos diferentes, pero con preocupaciones conexas que derivan del horizonte materialista histórico en el cual ambas obras se inscriben, las propuestas teóricas de Voloshinov y Pêcheux articulan la problemática de la ideología con la de la producción social de la significación y el sentido. De este modo, se abre un espacio de diálogo y confrontación que comenzamos a explorar ${ }^{2}$ desde una perspectiva que pone en foco a la categoría de sujeto: en ese sentido, interrogamos su estatuto a la vez que situamos su función en la economía teórica de ambos pensadores.

Nacido al calor de la polémica con el idealismo, el concepto de ideología conoció los "avatares del reconocimiento" propios de todo concepto que se mueve en un campo discursivo -el del marxismo- en donde la variación de las coyunturas y la reflexión y debate concomitantes operaron en favor de una productividad teórica no exenta de atascos y dificultades. Así, el concepto de ideología se presenta con lo que podríamos llamar un "ca- rácter complejo". Con ello no nos referimos solo a las múltiples maneras de entender y formular el concepto, sino al hecho de que, cuando se habla de "ideología" o de lo "ideológico" en el discurso marxista, se hace al menos con referencia a:

1. Un nivel o instancia de la vida social -más o menos determinado por otros niveles o instancias- que cumple algún papel en dicha vida social.

2. Una forma de captar, definir y explicar "lo que las cosas son" (ya sea a través de una concepción del mundo, representación, discurso) que en la mayor parte de los casos (a) se diferencia del conocimiento; (b) supone algún tipo de ilusión -a veces necesaria- que es producto del posicionamiento de quien sostiene y se sostiene en una ideología determinada: en ciertos casos, el carácter ilusorio es puesto en suspenso, pero señalando que los posicionamientos encontrados de los agentes suponen un abanico de discrepancias en sus posiciones ideológicas.

En lo que hace al primer punto, tanto Voloshinov como Pêcheux diluyen,

2 En el marco de nuestro trabajo colectivo, señalamos como antecedente de peso la intervención de Leandro Viterbo, que bajo el título "Althusser y Voloshinov: aproximaciones a un diálogo posible", fue presentada en las jornadas "Por el camino de Althusser. Marxismo y psicoanálisis. Legados y perspectivas" (Buenos Aires, 24 y 25 de agosto del 2009). 
a su manera, el esquematismo basesuperestructura en la medida en que, al empalmar la actividad ideológica a la producción de las significaciones no pueden sino diseminar dicha actividad en la totalidad social. Y aun cuando en Voloshinov la crítica de la tópica base-superestructura no se lleve hasta sus últimas consecuencias -ya que al pensar las distintas áreas especializadas de la actividad ideológica (ciencia, arte, religión) no deja de situarlas en el nivel de la superestructura-, el hecho de caracterizar a la conciencia en tanto que sígnica lo aleja de una de las flaquezas mayores de la tópica, es decir, aquella que termina considerando que el nivel de la superestructura no es un nivel práctico-material (lo que Althusser llamaba "la ideología de la ideología").

Sin dudas, las diferencias más notables en las posiciones de ambos autores se revelan en la consideración del segundo punto. Para avanzar en esta cuestión, interesa destacar que las condiciones de producción de los autores difieren en varios puntos sustanciales. En el caso de Pêcheux, la lectura de Althusser y la inscripción de su trabajo en un escenario que ya conocía el estructuralismo marca una primera distancia con el planteo de Voloshinov; otra está relacionada con que la realización de este último se inscribe en una época donde la problemática de la noción de ideología no estaba formalmente teorizada, y si lo estaba, era desde perspectivas harto diferentes: como el mismo Voloshinov señalara, "[...] en los estudios marxistas no existe todavía una definición completa y comúnmente aceptada de la realidad específica de los fenómenos ideológicos" (2009: 17).

En este sentido, vale detenerse en el siguiente detalle: La ideología alemana, el texto de Marx y Engels, fue publicado en ruso por primera vez en 1924. Si bien no es el objeto de nuestro trabajo, de este señalamiento puede inferirse que la inscripción de lo discursivo en lo ideológico resulta diferente en ambos autores a raíz del grado de desarrollo de la teoría de la ideología. Señala Ricoeur que, a partir de La ideología alemana, pueden seguirse dos lecturas, las cuales sin duda han tenido sus respectivos alcances. Por un lado, una vertiente estructuralista (a la cual asocia a Althusser), que toma del texto de Marx y Engels los aspectos que hacen a las estructuras despojadas de todo individuo: la relación entre realidad e ideología corresponde a la de la estructura y superestructura, no a la que se da entre individuo y conciencia. La segunda de las líneas considera la base real de la historia como el terreno donde individuos reales viven en condiciones históricamente definidas. Esta lectura pone de relieve el lugar de los hombres como las verdaderas víctimas de la división del trabajo y de la estructura de clases (Ricoeur 1989).

Podría aventurarse que la lectura de Voloshinov se encuentra más en sintonía con esta segunda vertiente, de manera que la concepción de los fe- 
nómenos de significación estará atravesada por un concepto de ideología en relación con los hombres reales y actuantes en la historia:

Donde el análisis lingüístico ve sólo palabras y las interrelaciones de sus factores abstractos (fonéticos, morfológicos, sintácticos, etc.), para la percepción artística viva y el análisis sociológico concreto se revelan relaciones entre personas, relaciones que el material verbal no hace más que reflejar y fijar. El discurso es el esqueleto que sólo adquiere una carne viviente en el proceso de la percepción creativa; en consecuencia, sólo el proceso de la comunicación viviente (Voloshinov 1999: 191, las cursivas son nuestras).

Volveremos sobre este punto en el último de los apartados de nuestro trabajo, dedicado a la cuestión del sujeto. Valga por el momento a modo de señalamiento respecto del concepto de ideología, la cual aparece como indiscernible de la práctica de sujetos reales en la historia.

Voloshinov define lo ideológico por su carácter sígnico; y en tanto que signo se modela en la situación comunicativa de acuerdo a su contenido y al acento valorativo que lo acompaña (Voloshinov 2009: 44). Es en el proceso de comunicación -que de ningún modo se realiza a título individual, sino por el contrario es informado según la configuración social específica y el momento histórico del que se trate- donde se entrecruzan esos distintos acentos. Ahora bien, tal como fuera señalado en párrafos anteriores, es de acuerdo a cómo Voloshinov se posiciona frente a la cuestión de la determinación que se observa que lo ideológico está marcado por la conflictividad que escinde lo social en el plano de la producción. La interacción comunicativa no está planteada bajo el precepto de una sociedad armónica: las fuerzas contradictorias que dinamizan el terreno económico se despliegan en la instancia de lo ideológico. A su vez, el carácter multiacentuado del signo le permite afirmar que el sentido nunca es estático -por el contrario, es móvil, dinámico- y desarrollar con mayor potencia la concepción marxista de lo social: si en todo signo ideológico se cruzan acentos diferenciales, y la operación de dominación consiste en presentarlo con un acento único, monoacentuarlo, el signo es la arena de la lucha de clases (2009: 47). Dicha formulación pone de relieve que el signo, lo ideológico, es una de las esferas específicas donde no solo se significa el mundo y la vivencia de la vida cotidiana, sino donde se despliega la conflictividad que define lo social, es decir, la lucha de clases.

Para dar cuenta de la concepción de Pêcheux sobre lo ideológico, debemos, necesariamente, hacer un rodeo para mencionar, aunque sea brevemente, las tesis althusserianas bajo las cuales se inscribe su proyecto. Señalemos en primer lugar que, a diferencia del contexto histórico donde escribe Voloshinov, la inserción del análisis del discurso como una región dentro del materialismo histórico supone la 
problematización de la determinación por la base que venimos de mencionar, pero con la ventaja de que en el caso de Pêcheux se realiza a la luz del concepto de sobredeterminación althusseriano, el cual considera la relativa autonomía y la eficacia específica de los fenómenos superestructurales.

Pêcheux, dentro de su objetivo declarado de "sentar las bases de la teoría materialista del discurso" (Pêcheux 2003: 157), realiza una explicación de ciertos conceptos tomados de la teoría de Althusser, interesante para deslindar su vínculo específico con el materialismo histórico. De manera general, retoma la problemática de los aparatos ideológicos de Estado (AIE) como terreno atravesado por la lucha de clases, para indicar que allí también se contribuye a la reproducción/transformación de las relaciones de producción. A partir de esto, se planteó un vínculo entre lo ideológico y las estructuras constitutivas de lo social. Lo ideológico se constituye en aparatos: se aprecia la distancia con la inscripción planteada por Voloshinov. Ahora bien, Pêcheux se encarga de especificar su lectura de Althusser: los AIE no realizan la ideología en general (una suerte de espíritu de época que se impone de manera homogénea en el conjunto de la sociedad) ni son tampoco el instrumento de una clase dominante que hace uso de ellos de manera consciente: constituyen el escenario donde esa dominación se realiza. Estos aparatos forman en cada sociedad un conjunto complejo, donde sus relaciones contradictorias dan existencia material a la lucha ideológica de clases y donde las clases mismas se constituyen en ciertos aspectos.

Las relaciones entre personas referidas por Voloshinov adquieren aquí un estatuto problemático: la teoría althusseriana explica, mediante el mecanismo de interpelación, los modos en que cada individuo es siempre-ya sujeto. Las formas de lo social anteceden a todo niño que viene al mundo y le reservan un lugar. A partir de ese emplazamiento inicial, el sujeto se inscribirá en el todo sobre la base de un reconocimiento -erróneo- de su propia autonomía, así como de un desconocimiento del proceso por el cual lo social le ha asignado una posición determinada. La ideología produce una red de "[...] verdades evidentes 'subjetivas' donde 'subjetivas' significa no 'que afectan al sujeto' sino 'en las que el sujeto se constituye'" (Pêcheux 2003: 164). El punto que Pêcheux retoma especialmente para insertar un análisis específico de lo discursivo es allí donde, casi al pasar, Althusser enuncia que esa evidencia espontánea del sujeto es análoga a la evidencia del significado. El poder para dar cuenta del principio de no conciencia que posee la conceptualización althusseriana derivada de su teoría de la interpelación en conjunto con la de los AIE es uno de los aspectos que Pêcheux señala con mayor énfasis: "se habla del sujeto y al sujeto antes de que el sujeto pueda decir 'Hablo'”' (Pêcheux 2003: 165). 
No obstante, y a partir de las diferencias apenas esbozadas aquí, es posible afirmar que en ambos autores la ideología aparece como un elemento central de lo social y que no está opuesta a la realidad como una distorsión que fuera posible de eliminar. Muy por el contrario, lo ideológico es integrante de la vida social, y en ambos posee un papel activo. Si bien respecto de Pêcheux puede decirse que el problema de la sobredeterminación había sido ya elaborado por Althusser, en Voloshinov también se advierte -por el simple intento de elaborar una filosofía marxista del lenguaje- que los fenómenos discursivos tienen su peso específico en la coyuntura social.

\section{Problemas de la dicotomía lengua/habla}

Tanto en Pêcheux como en Voloshinov es posible encontrar un posicionamiento crítico respecto a la distinción entre lengua y habla. En ambos hay señalamientos conducentes a considerar que tanto aquellas posiciones que -dicho brevemente- se basan en el sistema de la lengua, como aquellas que privilegian la expresión subjetiva, son, llegado cierto punto, complementarias y erróneas. En este sentido, la distinción lengua/habla comporta consecuencias que habrán de ser rechazadas, fundamentalmente en lo que concierne al último de los puntos por tratar en este trabajo: el lugar del sujeto. En la medida en que el privilegio de ninguno de los dos polos será satisfactorio para los autores, se analizarán sus esfuerzos para desarrollar nuevas construcciones teóricas, capaces de dar cuenta a la vez de los procesos discursivos e ideológicos, lo que implica introducir la cuestión de lo social y de lo histórico.

Pêcheux se preocupa por la dimensión epistemológica del abordaje de los problemas de la significación. En su proyecto de desarrollo de un análisis automático del discurso (procedimiento técnico-metodológico diseñado para leer un corpus discursivo), aparecen tres regiones dando cuenta del "cuadro epistemológico general" en el que se inscribe:

1. El materialismo histórico como teoría de las formaciones sociales y de sus transformaciones, incluida la teoría de las ideologías.

2. La lingüística como teoría de los mecanismos sintácticos y a la vez de los procesos de enunciación.

3. La teoría del discurso como teoría de la determinación histórica de los procesos semánticos.

Añadamos que esas tres regiones se ven, en cierta manera, atravesadas y articuladas por la referencia -que convendrá hacer explícita- a una teoría de la subjetividad (de naturaleza psicoanalítica) (Pêcheux 1978: 228).

Se observan dos cuestiones por tratar: en primer lugar, la importancia que adquiere para el autor la posibili- 
dad de situar la disciplina del análisis de discurso dentro de un cuadro epistemológico que le permita avanzar en la construcción de conocimiento científico; en segundo lugar, el hecho de colocar la lingüística en un territorio de articulación con otras regiones teóricas. Ambos aspectos están relacionados: en Pêcheux, la consideración de Saussure adquirirá matices críticos en relación con las consecuencias que derivan del planteo de la lingüística, pero no obstante el gesto de construcción de una disciplina científica será considerado como una ruptura en ciertos términos valorada.

En un texto publicado bajo el seudónimo de Thomas Herbert, ${ }^{3}$ Pêcheux afirma -en una frase con resonancias althusserianas- que "[...] toda ciencia es principalmente ciencia de la ideología de la cual se separa". En ese sentido, considera que Saussure produce una ruptura: "El principio de la subordinación de la significación al valor puede considerarse, en nuestra opinión, como el núcleo de la ruptura saussuriana" (Haroche, Henry y Pêcheux 1971: 96). A partir de la noción central de valor, de la fundación de la lengua como sistema y de lo que designan como el principio de unidad de la lengua, se evidencia un cambio radical tanto en el plano teórico como en el de la práctica del lingüista:
La gramática histórica estaba basada en las comparaciones entre elementos aislados pertenecientes a lenguas diferentes, supuestamente ligados por filiación histórica: la lingüística postsaussureana acuerda la prioridad a las operaciones de conmutación, comparaciones regladas, etc., dentro de una misma lengua, es decir al funcionamiento de las lenguas en relación consigo mismas en el cuadro de una lingüística general que es la teoría de ese funcionamiento (Haroche, Henry y Pêcheux 1971: 99).

Entonces, partiendo de esta aceptación en términos epistemológicos del gesto saussureano como una ruptura, es posible introducirse en los cuestionamientos a la separación entre lengua y habla. Muchas de dichas críticas apuntan, más que al mismo Saussure, a los desarrollos posteriores que se hicieron aplicando o expandiendo los principios explícitos e implícitos de la lingüística tal como aparece en el Curso de 1915. No obstante, el núcleo central de señalamientos a la dicotomía tiene que ver con el lugar asignado -por acción u omisión- al habla: mientras que la lengua expulsa al sujeto (lo cual puede pensarse que es valorado por Pêcheux), el problema radica en la reintroducción del sujeto del lado del habla. De esta manera, aparecen dos tipos de problemas conexos: los que refieren a la semántica y los relativos al sujeto hablante.

3 Nos referimos al artículo“Notas para una teoría general de las ideologías”, publicado en el número de Cahiers pour l'Analyse, el año 1968. 
[...] la oposición lengua/habla, históricamente necesaria para la constitución de la lingüística, va de la mano con cierta ingenuidad de Saussure con respecto a la sociología, de hecho bastante explicable, porque los mismos sociólogos contemporáneos de Saussure la compartían a menudo: esta ingenuidad reposaba sobre una ideología individualista y subjetivista de la "creación" [...]. ¿No encontramos aún esta misma ideología en la idea de Jakobson según la cual, del nivel del fonema al del encadenamiento de las frases, se pasaría de la limitación lingüística a esta libertad por la cual el sujeto hablante dice "eso que jamás se escuchará dos veces"? El par libertad/ limitación o, si se prefiere, creativi$\mathrm{dad} /$ sistema tiene las propiedades circulares de un par ideológico, en la medida que cada uno de los términos en presencia presupone al otro: la creatividad supone en efecto la existencia de un sistema que ella pudiera hacer estallar, y todo sistema no es sino el efecto de una creatividad anterior. La noción de sistema [...] aparece entonces como el complemento indispensable de la creatividad, dentro del campo del "lenguaje": en otros términos, la oposición lengua/habla introducida por Saussure se encuentra repetida analógicamente en el habla bajo la forma de la oposición sistema/creatividad (resultante de la transposición de oposiciones tales como paradigma/ sintagma, sincronía/diacronía, etc.) (Haroche, Henry y Pêcheux 1971: 98).

No se afirma que el corte lengua/ habla no tenga valor epistemológico, sino que el obstáculo proviene de lo que queda del lado del habla. Por la subordinación saussureana de la significación al valor (en la lengua), la cuestión de la semántica queda sin abordar, y por el lado del habla se termina reintroduciendo un sujeto hablante como fuente de sentido. Este problema encuentra diferentes vías de solución. Más allá de la expulsión de la semántica del dominio de la lingüística -y del consiguiente planteo de una independencia de semántica y sintaxis-, otra posibilidad ha sido la inclusión completa de la semántica dentro de la lingüística, concepción que, señala Pêcheux, está paradójicamente vinculada a la anterior, ya que postula de manera implícita que el sentido es un hecho de lengua y por ello el sujeto hablante permanece neutro e ideal. Una tercera posibilidad consiste en pensar que determinados hechos semánticos son susceptibles de ser estudiados por la lingüística, mientras que otros no. Dentro de este grupo, detecta también una partición. Por una parte, la propuesta de Benveniste, en su separación entre sentido (intralingüístico) y referencia (extralingüística), si bien inaugura la posibilidad de analizar aspectos semánticos, permanece presa de la ilusión subjetiva del habla (Pêcheux 1978: 344 y ss.). Por la otra, aparece su propio encuadre, que será desarrollado con más detalle en el apartado próximo.

[...] lo que se designa hoy bajo el nombre de semántica no depende sino parcialmente de un abordaje lingüístico. [...] En esas condiciones, la semántica 
(en tanto que teoría de las regiones dejadas fuera del campo de aplicación de los conceptos y de la práctica de los lingüistas) supone un cambio de terreno o de perspectiva (Haroche, Henry y Pêcheux 1971: 94).

Este "cambio de terreno" supondrá la introducción de nociones provenientes del materialismo histórico (ya que la producción de sentido se inscribe dentro de los procesos ideológicos) y en cierta medida del psicoanálisis (en el cuestionamiento al sujeto de conciencia).

Es posible entonces sostener que la cuestión se dirime en los términos de cómo pensar y cómo dar cuenta del lenguaje: no como una función de la expresión, sino como sistema. En este sentido, la apuesta de Pêcheux puede ser vista en una doble dirección: por un lado, intenta, en su inscripción en el aparato conceptual althusseriano, dar cuenta de la producción del sentido (cuestión que parecería perderse en la exclusión del habla). Por el otro, y en consonancia con lo planteado hasta aquí, se realiza un intento por "desubjetivizar" ese mismo proceso: si en la oposición lengua/habla reingresa el problema del sujeto de voluntad y conciencia que Saussure pareciera haber querido descartar ("El habla, como uso de la lengua, aparece como un camino de la libertad humana" [Pêcheux 1978: 33]), adquiere su lugar propio un planteo que dé cuenta de lo discursivo en articulación con una concepción de lo ideológico en términos de una mira- da centrada en los procesos históricos a-subjetivos de producción discursiva.

En Voloshinov, en cambio, no hay una recuperación semejante de la labor teórica de Saussure, a quien ubica como paradigma del "objetivismo abstracto", una de las dos vertientes que, junto con lo que llama el "subjetivismo individualista", han equivocado su rumbo al pensar el lenguaje. En la discusión que Voloshinov entabla con dicho "objetivismo abstracto" se evidencia el tipo de problemas que el autor detecta respecto de dicha postura. Podría leerse su crítica en el horizonte del materialismo histórico clásico, desde la frase de Marx según la cual "los hombres hacen la historia pero en condiciones independientes de su voluntad". Sin embargo, en el curso de su desarrollo el énfasis no recae tanto sobre tales condiciones, sino más bien, y en consonancia con lo planteado respecto de la noción de ideología, se privilegia el lugar de la creatividad social. A la inversa, podría decirse que Pêcheux le presta más atención a las condiciones que a la agencia.

No obstante, también puede pensarse que las condiciones, para Voloshinov, se articulan conceptualmente de otro modo: las determinaciones sociohistóricas aparecen como horizontes donde la producción sígnica se inscribe. Existen horizontes inmediatos (la situación concreta de enunciación) y otros más lejanos y generales ("las relaciones sociales más duraderas y profundas en las cuales el 
hablante participa" [Voloshinov 2009: 139]). En Pêcheux, aquello que proporciona la mayor inmediatez en la determinación de un proceso de discurso son las formaciones imaginarias desarrolladas en 1969; concepto que, en la revisión crítica de 1975, habrá de objetar en la medida en que, según su criterio, dejaba el camino abierto para recaer en una perspectiva sociolingüística donde el hablante empírico y la posición imaginaria se identificasen equivocadamente. En Voloshinov, los contextos cercanos y lejanos intervienen en la producción discursiva en dos sentidos: primero, el hablante se orienta hacia ellos; y luego, la conciencia individual está ya estructurada en términos sígnicos, lo cual es equivalente a decir ideológicos y, por consiguiente, sociales.

Tal como insinuamos más arriba, la doble crítica que realiza Voloshinov -a lo que designa como objetivismo abstracto y subjetivismo individualistapuede ser leída en los términos de un mismo señalamiento:

El objetivismo abstracto, al considerar el sistema de la lengua como lo único importante para el análisis de los fenómenos lingüísticos, rechaza el acto discursivo -la enunciación- como acto individual. [...] En ello consiste el proton pseudos del objetivismo abstracto. El subjetivismo individualista considera precisamente el acto discursivo, o la enunciación como lo único que importa. Pero también esta corriente define este acto como individual y por tanto trata de explicarlo desde las con- diciones de vida individual y psíquica de la persona. Éste es su propio proton pseudos (Voloshinov 2009: 132).

En tanto el gesto que crea la lengua como objeto de conocimiento implica una expulsión del sujeto al terreno del habla, y esta queda como el lugar de la libertad creadora, se ve que, en ese sentido, ambos enfoques dejan abierto el camino a la individualidad inmediata. Se entiende entonces por qué el autor señala que "[...] uno de los errores más profundos del objetivismo abstracto es la ruptura entre la lengua y su capacidad ideológica" (Voloshinov 2009: 113):

En realidad, un acto discursivo o, más exactamente, su producto el enunciado, no puede ser reconocido como fenómeno individual en el sentido exacto de la palabra ni puede ser explicado a partir de las condiciones psicológico-individuales o psico-fisiológicas del sujeto hablante. El enunciado tiene carácter sociológico (Voloshinov 2009: 132).

Esta constatación, al tiempo que descarta los fundamentos explícitos e implícitos que sostienen a las mayores corrientes de pensamiento sobre el lenguaje, le permite introducir su aporte a esta área disciplinaria desde el cruce con la perspectiva marxista.

\section{Construcción de un nuevo objeto: Discurso}

La crítica a la dicotomía lengua/habla se inscribe, en ambos autores, en la necesidad de pensar lo que el modelo 
de la lengua no permite considerar: lo discursivo. ${ }^{4}$ Para Pêcheux (más fiel en este sentido al legado saussuriano), la lengua constituye en todo caso la base sobre la cual se genera el proceso discursivo, pero no se puede estudiar dicho proceso sin la articulación de los aportes lingüísticos con la teoría de la ideología althusseriana, con la teoría del sujeto del psicoanálisis y con una nueva semántica específicamente discursiva. Para Voloshinov -en esto, en ruptura radical con los presupuestos saussurianos- se torna necesario pensar que el objeto real del estudio del lenguaje no es la estructura de la lengua, sino la interacción discursiva.

Debemos entonces considerar comparativamente el lugar que lo discursivo ocupa en los planteos de ambos, tanto en relación con la crítica a la distinción lengua/habla como en lo tocante a la inscripción respecto de la ideología.

Así, en el caso de Voloshinov observamos que su concepción del signo ideológico (Voloshinov 2009: 25-36) permite, ante todo, fundar la materialidad del signo excluyendo la problemática de la conciencia, en la medida en que toda conciencia no es sino un entrecruzamiento de signos: "La conciencia sólo deviene conciencia al llenarse de un contenido ideológico, es decir sígni- co, y por ende, sólo en el proceso de interacción social" (Voloshinov 2009: 29).

De lo anterior se desprende que las diversas formas de la ideología son siempre ya operantes en la organización social, de la cual la conciencia es producto. Basándose en la concepción de la "psicología social" de Plejánov -que se entiende como el "eslabón transitivo entre una formación político-social y una ideología en el sentido restringido (la ciencia, el arte, etc.)" (Voloshinov 2009: 41)- asumirá que la forma material de esta "psicología" es la interacción discursiva:

La psicología social es precisamente aquel medio ambiente que, compuesto de las actuaciones discursivas más variadas, abarca multilateralmente todas las formas y aspectos de la creación ideológica (Voloshinov 2009: 41).

Como se sabe, este "medio ambiente" no conforma un escenario de apacible armonía, sino que es el ámbito de la lucha ideológica: "[...] cada palabra es una pequeña arena de cruce y lucha de los acentos sociales de diversas orientaciones" (Voloshinov 2009: 73). La producción discursiva es así inscrita en una situación de interacción, cuya forma privilegiada es el diálogo (2009: 151-153). Es decir, ni sistema abstracto, ni acto psicofísico individual, el discurso -el acto de enunciación y

4 Utilizamos el término provisoriamente y en un sentido general que será luego necesario precisar atendiendo a las particularidades de los planteos de cada autor. 
las formas del enunciado en las que se plasma- debe analizarse bajo la lógica de la comunicación dialógica, situada histórica y socialmente, y emplazada en la lucha de clases.

Pero el diálogo puede ser comprendido extensivamente, no solamente como la comunicación verbal directa $\mathrm{y}$ oral de las personas presentes, sino como toda comunicación discursiva del tipo que sea. [...] Así pues, una actuación discursiva participa en una discusión ideológica a gran escala: responde a algo, algo rechaza, algo está afirmando, anticipa las posibles respuestas y refutaciones, busca apoyo, etcétera.

Todo enunciado, por más terminado e importante que fuese en sí mismo, es tan sólo un momento en la comunicación discursiva continua (cotidiana, literaria, cognoscitiva, política). Pero además, este intercambio discursivo es, a su vez, tan sólo un momento de un continuo y multilateral proceso generativo de un colectivo social dado (Voloshinov 2009: 151-153).

De manera peculiar, y en consonancia con la lectura de los textos de Marx que se deslindó más arriba, se advierte que el objeto discurso adquiere matices específicos en Voloshinov. Por un lado, aparece como producto concreto de los hombres reales y actuantes en la historia; por el otro, como materialidad significante, constituyente de un acervo de enunciados ya dichos que alimenta cada producción discursiva -y entonces también la lucha ideológica- de manera ineludible. Examinado desde el primer punto de vista, cada enunciado puede remitirse a personas presentes en un diálogo real, y de aquí la acusación de Pêcheux respecto de tratarse de una variante de la sociolingüística (Gadet y Pêcheux 1984: 104). No recae, por ello, en un principio de sujeto-consciente, en la medida en que la conciencia, como se dijo, está ideológicamente estructurada por estar constituida solo por signos.

Desde el segundo punto de vista, la concepción de discurso aparece más cercana a lo que Pêcheux designa como las condiciones de producción de un discurso. Lo discursivo funciona en Voloshinov en un doble nivel: el discurso de los sujetos y un discurso del colectivo anónimo. En ambos se conserva lo esencial de la crítica al subjetivismo individualista y al objetivismo abstracto: en el nivel de las conversaciones cotidianas, la conciencia del sujeto hablante acusa intervención de lo social: en el nivel del colectivo abstracto, el anclaje histórico impide el desprendimiento de lo discursivo como un objeto independiente de las condiciones históricas de emergencia.

Pasemos ahora a la concepción que elabora Pêcheux del nuevo objeto discurso. Pêcheux señala:

La progresiva constitución del análisis de discurso como práctica específica (con sus propios conceptos, sus problemáticas y sus procedimientos) tiende a transformar esta situación, al tematizar el objeto 'discursividad' 
como objeto teórico de frontera, en contacto efectivo con investigaciones lingüísticas por un lado, y socio-históricas por el otro (Pêcheux 1984).

Esa frontera donde emerge lo discursivo supone la posibilidad teórica de dar cuenta de manifestaciones verbales históricamente determinadas, marcando distancia respecto de la confusión entre discurso y habla como terreno de la libertad subjetiva (lo que conduciría a una lingüística del habla), así como respecto de lo discursivo como un complemento para una posición dada en la estructura social (conducente a la sociolingüística).

Dar cuenta del discurso supone, respecto de la lengua, tomarla como el sistema que provee elementos seleccionables y recombinables (condición de posibilidad para el proceso) sobre la cual se despliega el proceso productivo, el que será resultado de tales operaciones sobre el sistema lingüístico a partir de condiciones de producción dadas (resultado de procesos discursivos sedimentados) en un estado histórico determinado (Pêcheux 1969). Este proceso no se lleva adelante en general, sino de manera situada en el concierto de las formaciones ideológicas y discursivas de una formación social determinada. En Pêcheux, lo ideológico y lo discursivo no coinciden, dado que, según el autor, identificar los elementos sería concebir lo ideológico de manera idealista, como esfera de los discursos y las ideas (Pêcheux 1975:
233). Lo discursivo es, por el contrario, una de las manifestaciones materiales de la ideología.

A su vez, hablar tanto de "lo discursivo" como de "lo ideológico" supone inscribirlos dentro de "formaciones", lo cual señala, por un lado, la necesaria (y conflictiva) conexión de lo discursivo vía lo ideológico con el todo de la formación social, así como la intervención de reglas específicas para cada nivel. La producción de discursos, entonces, está marcada no solo por las reglas estrictamente lingüísticas. Podría decirse que estas llegan hasta pasar a un segundo plano, en la medida en que las reglas de formación de discursos vienen orientadas por la inscripción de cada uno de ellos dentro de una o más formaciones discursivas. En el gesto de Pêcheux se advierte, entonces, un doble señalamiento:

- Lo discursivo como una de las formas materiales de manifestación de lo ideológico (no hay coincidencia entre ideología y discurso).

- Existen reglas objetivas históricamente determinadas específicas para la producción de discursos (plantear lo discursivo implica recortar otras unidades, como las formaciones discursivas).

A modo de recapitulación y aún a riesgo de resultar repetitivos, señalemos los puntos en común entre ambos autores. Se trata de concepciones de discurso emergentes de: 
A. una crítica a la dicotomía lengua/ habla;

B. una articulación con la inscripción de lo discursivo en lo ideológico;

C. un cuestionamiento al sujeto como hablante (en tanto que fuente originaria de la significación o el sentido);

De lo cual se derivan, como puntos centrales, algunas cuestiones comunes que vamos a señalar:

A. La necesidad metodológica de emplazar cada discurso puntual en un concierto discursivo más amplio: "es imposible analizar un discurso como un texto" (Pêcheux 1969: 44), y, como corolario, la ausencia de principio o fin del proceso de generación discursiva.

B. La necesaria complejización de la relación entre los fenómenos de la base económica y los procesos ideológicos: en ambos, lo superestructural aparece como un todo con áreas específicas donde las transformaciones de la base económica adquieren manifestación a partir de sutiles y complejos mecanismos regidos por leyes propias de lo ideológico y lo discursivo. En Pêcheux, las diversas formaciones -ideológicas y discursivas, a su vez regionales y de clase- dan cuenta del concepto de sobredeterminación en Althusser. En Voloshinov, la relación entre las formas de la interacción discursiva y los contenidos o temas del discurso como algo para ser estudiado, supone un funcionamiento complejo de la instancia superestructural respecto de la base. ${ }^{5}$

C. El lugar de lo discursivo como materialización sígnica de la lucha de clases: Todo enunciado aparece en el interior de una relación de fuerzas entre clases o fracciones de clase en disputa. En Voloshinov, se manifiesta en el carácter reflexivo y refractario del signo; en Pêcheux en los procesos de metaforización, paráfrasis y bloqueo metafóricos.

D. El carácter performativo del discurso: En Pêcheux se advierte en el hecho de que un discurso efectivo pasa a formar parte de las condiciones de producción de los siguientes. En Voloshinov, en su concepto de diálogo comprendido de manera extendida.

Entre las diferencias, señalemos que en Voloshinov signo e ideología aparecen como coextensivos, mientras que en Pêcheux las formaciones discursivas son uno de los aspectos de las formaciones ideológicas. Por otro lado, las consecuencias derivadas de un posicionamiento diferente dentro del propio marxismo conducen a los autores a realizar más énfasis en los hombres reales o en las estructuras objetivas. Asimismo, el esfuerzo en

5 Cf., al respecto, el ejemplo del hombre superfluo (2009: 38). 
Pêcheux por recortar formaciones discursivas -si bien señala el error de pretender establecer para una sociedad dada los límites precisos entre ellasevidencia su interés por desarrollar una metodología precisa de análisis de discurso, cuestión que no aparece en Voloshinov como objetivo central. En este último, el señalamiento acerca de los estratos de la ideología cotidiana y de las áreas de creatividad ideológica constituye más bien una constatación general de los modos de darse de lo sígnico. ${ }^{6}$

\section{Implicancias respecto de la noción de sujeto y complejidades para el análisis de discurso}

La noción de sujeto que puede rastrearse en ambos autores no solo es producto de las singulares articulaciones que cada uno de ellos realiza en torno al problema de la base, la ideología y el discurso; responde también a cómo hacen jugar el entrecruzamiento entre marxismo y psicoanálisis. Sin duda, los horizontes desde los cuales cada uno de los autores interroga las obras freudianas son disímiles: en el caso de Voloshinov, contemporáneo con aquel, la polémica se inscribe en dos vectores: en oposición al problema de la conciencia, acuerda con Freud; pero en su polémica contra el subjetivismo, lo ubica como un referente entre otros de la psicología subjetivista: ${ }^{7}$ en la recuperación que realiza Pêcheux, en cambio, pueden identificarse, como ya se ha señalado, las huellas de las lecturas de Althusser y Lacan, entre otros.

Por otra parte, aun cuando en Voloshinov no hay una preocupación por formular una teoría formal del sujeto, cabría preguntarse si en sus formulaciones no está operando una determinada conceptualización en estado práctico. De esta manera, el análisis se desplegará en dos direcciones: por un lado, sobre su lectura de Freud se observa que es poco lo que puede extraer de los contundentes planteos freudianos; así, por ejemplo, frente a la noción de inconsciente dirá que es producto de lo social, por lo tanto propondrá denominarlo "conciencia no oficial" (cf. Voloshinov 1999: 157 y ss.) y para dar cuenta del contenido de la

6 Cf. en Voloshinov los estratos de la ideología cotidiana (2009: 146 y ss.), donde la descripción no arroja elementos para un recorte sistemático de las áreas de creatividad ideológica sino que se trata más bien de una constatación de regiones generales y más o menos imprecisas.

7 Las críticas realizadas al "freudismo" están vinculadas a cierta concepción, hoy del sentido común, que distingue en los textos de Freud un enfoque más individual de otro más social; así es que Voloshinov apunta a cuestionar esta impronta individualista, a atacar las metáforas biologicistas con las que Freud intenta dar cuenta del juego de fuerzas psíquicas que dinamizan el terreno inconsciente, así como la técnica de la asociación libre con referencia al estatuto y la relación entre la conciencia y la palabra (en particular, cf. 2009: 157 y ss.). 
psique afirmará que es ideológico "de cabo a rabo". En segundo término, en relación con el proceso comunicativo establecerá una distinción entre signo y señal, donde argumenta que, dado que una señal no está atravesada por diversos acentos valorativos (no es del orden de lo ideológico), de lo que se trata es de reconocerla; mientras que el signo ideológico supone una actividad de comprensión:

[...] la tarea de la comprensión, en general, no se reduce al reconocimiento de una forma aplicada, sino a su comprensión precisamente en un contexto dado y concreto, a la comprensión de su significación en un enunciado dado, es decir, a la comprensión de su novedad pero no al reconocimiento de su identidad (Voloshinov 2009: 109).

La comprensión del signo implica, en los participantes del proceso comunicacional, la vivencia misma de su significación ("En la vida real, nosotros jamás pronunciamos ni oímos palabras, sino que oímos la verdad o la mentira, lo bueno, lo malo, lo importante o lo nimio, lo agradable o lo desagradable" [Voloshinov 2009: 112]). Es así como en una situación comunicativa, situada histórica y socialmente, la participación subjetiva se organiza en función a la multiplicidad de valoraciones que desde los actores intervinientes pueden tensionar el sentido de un discurso.

En Pêcheux, en cambio, la preocupación sobre la cuestión del sujeto es explícita: ¿cómo conceptualizar el agenciamiento subjetivo sin recaer en el sujeto cartesiano? A este respecto, tal como hemos dicho, su inscripción en la propuesta althusseriana resulta por demás enriquecedora, ya que al recuperar la noción de interpelación ideológica ubica en el centro de estos planteos la premisa fundamental de la constitución subjetiva descentrada. Aquí se tornan fecundas las nociones de "olvidos", a partir de las cuales se pone de relieve que toda producción discursiva se fundamenta en dos fallas: una vinculada al registro lacaniano de lo real, donde Pêcheux señala que notodo puede ser significado (olvido $\mathrm{N}^{\circ}$ 1) y que este desconocimiento por parte del sujeto es el que funda el juego de la producción discursiva; la segunda, de orden imaginario (olvido $\mathrm{N}^{\circ}$ 2), permite el análisis de los procesos discursivos concretos que se realizan a través de la identificación, necesaria e ilusoria a la vez, del sujeto a un yo fuente del sentido (Pêcheux 1978: 240 y ss). Así, "lo real del lenguaje" (Pêcheux y Gadet 1984: 48-51) pone de relieve un cierto vacío a partir del cual se configura no solo el enunciador sino también las superficies lingüísticas de su realización en una situación concreta.

En un artículo de 1984, Pêcheux focaliza en la cuestión del sujeto y discute mayormente con la psicología, mostrando un desplazamiento respecto del texto Hacia un análisis automático..., donde el énfasis estaba más bien pues- 
to en la articulación de la lingüística con premisas del materialismo histórico en un intento de dar cabida a la determinación material de los hechos de discurso. Si bien en ese entonces aparece la crítica al sujeto fuente de sentido, en la década de 1980 el foco se pone más de lleno sobre la cuestión del sujeto, entendiendo esta problemática como el correlato teórico inmediato a un encuadre del análisis de discurso que dé batalla tanto al cogito cartesiano como a la triple forclusión que detecta en las llamadas disciplinas de la interpretación en torno al hecho de que haya la historia, la lengua, el inconsciente (Pêcheux 1984).

Sostenemos que el modo en que ambos autores resuelven, explícita o implícitamente, el lugar del hablante extrema las diferencias entre ambos. En un caso, puede afirmarse que las consecuencias de los planteos de Voloshinov son dobles ya que se torna confuso delimitar cómo y desde qué lugar los sujetos intervienen en la interacción discursiva: si lo que caracteriza la intervención subjetiva está vinculada con la vivencia significativa del signo, y si, además, el discurso puede representarlo todo, entonces, el análisis del discurso se realiza, en términos althusserianos, desde y sobre lo imaginario. En la apuesta teórica de Pêcheux por realizar una teoría no subjetiva del discurso queda por pensar cómo analizar la producción de las significaciones sociales bajo el privilegio del principio saussureano del valor.

\section{A modo de cierre}

A lo largo del trabajo pusimos en diálogo los planteos de Michel Pêcheux y Valentin Voloshinov en torno de la emergencia de lo discursivo como un nuevo objeto de análisis. Esta emergencia mostró particularidades en relación con el lugar de su emplazamiento teórico pero también respecto de las consecuencias de asumir el nuevo planteo.

En el primer caso, el emplazamiento de lo discursivo se sitúa, por un lado, en discusión con la lingüística derivada de Saussure, sobre todo en relación con la separación lengua/ habla. Por otra parte, pensar el fenómeno del discurso implica dar cuenta de los modos en que las enunciaciones particulares se inscriben en el terreno sociohistórico, de allí el interés por las premisas del materialismo histórico, fundamentalmente en la problemática de la ideología y de la relación entre la base y la superestructura.

Respecto de las consecuencias teóricas, la construcción del concepto de discurso acarrea consecuencias en relación con las formas de pensar el sujeto: en ambos es posible dar con una crítica a los postulados modernos de un sujeto centrado en el sistema percepción-conciencia, el cual sería fuente del sentido de sus enunciaciones.

El recorrido propuesto permitió dejar sentadas áreas de problematización sobre las cuales no puede aún afirmarse que existan respuestas definitivas. 


\section{Bibliografía}

Althusser, L. (1979). Ideología y aparatos ideológicos de Estado. Buenos Aires: Nueva Visión.

—. (1969 [1965]). "Marxismo y humanismo". "Contradicción y sobredeterminación". La revolución teórica de Marx. México D. F.: Siglo XXI.

Gadet, F. y M. PÊcheux (1984). La lengua de nunca acabar. México D. F.: Fondo de Cultura Económica.

Haroche, C.; Henry, P. y M. Pêcheux (1971). "La sémantique et la coupure saussurienne: Langue, langage, discours". Langages. Año 6, núm. 24. $<$ http://www.persee.fr/web/revues/ home/prescript/article/lgge_0458726x_1971_num_6_24_2608>.

PêCheux, M. (2003). “El mecanismo del reconocimiento ideológico", en ŽızeK, S. (comp.). Ideología. Un mapa de la cuestión. Buenos Aires: Fondo de Cultura Económica.

—. (octubre de 1984). "Sur les contextes épistémologiques de l'analyse de discours". Mots 9. <http://www.persee.fr/web/revues/home/prescript/ article/mots_0243-6450_1984_ num_9_1_1160)>.

-. (1978). Hacia un análisis automático del discurso. Madrid: Gredos.

—. (1975). Les vérités de La Palice. París: Maspero.

—. (1969). Analyse automatique du discours. París: Dunod.

Ricoeur, Paul (1989). Ideología y utopía. Barcelona: Gedisa.

Voloshinov, V. (2009). El marxismo y la filosofía del lenguaje. Buenos Aires: Godot.

—. (1999 [1927]) Freudismo. Un bosquejo crítico. Buenos Aires: Paidós. 